\title{
Short-pulse Er:YAG laser increases bond strength of composite resin to sound and eroded dentin
}

\author{
Maria Cecília Pereira Cersosimo \\ Adriana Bona Matos \\ Roberta Souza D'Almeida Couto \\ Márcia Martins Marques \\ Patricia Moreira de Freitas
}




\title{
Short-pulse Er:YAG laser increases bond strength of composite resin to sound and eroded dentin
}

\author{
Maria Cecília Pereira Cersosimo, ${ }^{*}$ Adriana Bona Matos, Roberta Souza D’Almeida Couto, \\ Márcia Martins Marques, and Patricia Moreira de Freitas \\ University of Sao Paulo, Department of Operative Dentistry, School of Dentistry, Av Prof. Lineu Prestes, 2227, 05508-000, Sao Paulo, SP, Brazil
}

\begin{abstract}
This study evaluated the influence of the irradiation with a short-pulse Er:YAG laser on the adhesion of composite resin to sound and eroded dentin (SD and ED). Forty-six samples of occlusal dentine, obtained from human molars, had half of their surface protected, while the other half was submitted to erosive cycles. Afterward, 23 samples were irradiated with Er:YAG laser, resulting in four experimental groups: SD, sound irradiated dentine (SID_Er:YAG, $50 \mu \mathrm{s}, 2 \mathrm{~Hz}, 80 \mathrm{~mJ}$, and $12.6 \mathrm{~J} / \mathrm{cm}^{2}$ ), ED, and eroded irradiated dentin (EID-erosion + Er:YAG laser). A self-etching adhesive system was used, and then cylinders of composite resin were prepared. A microshear bond strength test was performed after $24 \mathrm{~h}$ storage $(n=20)$. The morphology of SD and ED, with or without Er:YAG laser irradiation, was evaluated under scanning electron microscopy $(n=3)$. Bond strength values (MPa) were subjected to analysis of variance followed by Tukey's test. Statistically significant differences were found among the experimental groups: SD (9.76 $\pm 3.39 \mathrm{~B}), \operatorname{SID}(12.77 \pm 5.09 \mathrm{~A})$, ED $(5.12 \pm 1.72 \mathrm{D})$, and EID $(7.62 \pm 3.39 \mathrm{C})$. Even though erosion reduces the adhesion to dentin, the surface irradiation with a short-pulse Er:YAG laser increases adhesion to both ED and SD. ๑ 2016 Society of Photo-Optical Instrumentation Engineers (SPIE) [DOI: 10.1117/1.JBO.21.4.048001]
\end{abstract}

Keywords: bond strength; erosion; Er:YAG; human dentin; short pulse laser.

Paper 160057R received Jan. 28, 2016; accepted for publication Mar. 24, 2016; published online Apr. 18, 2016.

\section{Introduction}

Dental erosion has been a major concern of dentists, as its prevalence has increased significantly in the population, even among children and youths. ${ }^{1-3}$

The etiology of tooth erosion is multifactorial, and after diagnosis, measures must be taken to prevent its progression or, regarding severe lesions with great surface loss, to restore the teeth's function or aesthetics, and/or treat dentin hypersensitivity. ${ }^{4}$ When tooth restoration becomes necessary, a direct restorative technique with composite resin is considered a useful conservative procedure. $^{4,5}$

However, the eroded dentin (ED) is a challenging substrate to bond to. ${ }^{5,6}$ The dissolution of hydroxyapatite crystals by erosion causes the exposure of long collagen fibrils and the increase of the inner diameter of dentinal tubules. ${ }^{7}$ This fibril network is very resistant to mechanical impact, such as brushing, ${ }^{8}$ and can compromise the permeability of adhesive systems and hybrid layer formation. Consequently, to reach a greater bond strength, it is necessary to prepare the tooth surface before the use of adhesive systems ${ }^{5,9}$ in order to selectively remove the outer demineralized dentin.

Techniques to treat the ED surface prior to bonding procedures have been investigated, ${ }^{5,9}$ and high-power lasers have been recently reported for that purpose..$^{10}$ As the wavelength of the Er: YAG laser $(\lambda=2.94 \mu \mathrm{m})$ coincides with the absorption band of water and hydroxyapatite, specific parameters can conservatively remove dental hard tissue (ablation process) and promote the selective removal of carious dentin, microbial reduction, and

*Address all correspondence to: Maria Cecília Pereira Cersosimo, E-mail: maria.cersosimo@usp.br pretreatment (prior to adhesive procedures) of the tooth surface. $^{11-14}$

Most studies on the use of Er:YAG lasers for tooth preparation have used equipment with a pulse width in the range from 100 to $350 \mu$ s. However, there is already commercially available equipment with shorter pulse widths that are reported to cause less or no thermal damage to the tooth surface. ${ }^{15-17}$ Although there are many studies on the use of Er:YAG laser on dentin prior to restorative procedures, ${ }^{12,13,16,18-22}$ little is known about its effect on ED. ${ }^{10}$ To date, no studies about the effects of short pulse Er:YAG laser on the bond strength of ED to composite resin have been conducted.

Given the information above, this in vitro study aimed to evaluate the effect of the dentin surface's (sound and eroded) irradiation with a short pulse width Er:YAG laser on the adhesion to composite resin.

\section{Materials and Methods}

\subsection{Ethical Aspects}

This study protocol was approved by the Committee of Ethics on Research of the School of Dentistry of the University of Sao Paulo (Protocol n. 944.762). The authors declare no conflicts of interest on this research.

\subsection{Sample Preparation}

Forty-six recently extracted third human molars were used.

Six dentin discs, obtained from six teeth, were used to compose the samples for the scanning electron microscope (SEM)

$1083-3668 / 2016 / \$ 25.00$ @ 2016 SPIE 
test. For this purpose, discs were cut in the middle, and 12 hemidiscs of dentin were obtained $(n=3)$. The remaining 40 teeth were also cut in discs, and 80 hemi-discs of dentin were obtained and used for bond strength analysis $(n=20)$ (Table 1). They were stored in chloramine $\mathrm{T}$ solution at $0.5 \%$, under refrigeration $\left(4^{\circ} \mathrm{C}\right)$ until the beginning of the experiment (up to three months). Experimental groups were divided according to the factors of substrate and surface treatment and were evaluated by bond strength and SEM.

The occlusal enamel was removed with a low-speed diamond saw (Isomet 1000, Buehler Ltd., Lake Buff, Illinois), to obtain dentine discs that were included on acrylic resin (JET Clássico, Sao Paulo, SP, Brazil). Dentine was polished (Ecomet 3, Buehler Ltd.) with 120, 240, 400, and 600-grit silicon carbide sandpaper discs (1 min each).

A fragment of an adhesive tape (Scotch 3M, 3M ESPE, Americana, SP, Brazil) of $\sim 3 \times 3 \mathrm{~mm}$ was placed on the occlusal surface of each disc, protecting the area that corresponded to the sound dentin (SD) (not exposed to erosive cycling) to delimit the SD area. The samples were then individually submitted to erosive cycling, consisting of the immersion of the samples in $10 \mathrm{~mL}$ of $0.05 \mathrm{M}$ citric acid solution (Merck Dermstadt, Germany), $\mathrm{pH} 2.3$, six times per day (10 min each), for five days. ${ }^{23}$ Between each immersion in acid solution, with an interval of $1 \mathrm{~h}$ each, and during the remaining time of the day, the specimens were stored in $10 \mathrm{~mL}$ of remineralizing solution $\left(\mathrm{H}_{3} \mathrm{PO}_{4} 4.8 \mathrm{mM}, \mathrm{KCl} 20: 10 \mathrm{mM}, \mathrm{Na}_{2} \mathrm{CO}_{3} 11.90 \mathrm{mM}\right.$, $\mathrm{CaCl}_{2} 1.98 \mathrm{mM}, \mathrm{pH}$ 6.7). ${ }^{24}$ The solutions were renewed daily at the beginning of the experiment, and the $\mathrm{pH}$ value of all solutions was checked at the beginning and end of each experimental day.

\subsection{Surface Treatment with the Er:YAG Laser}

After the erosive challenge, the adhesive tape was removed from the dentin samples' surfaces, exposing the SD area. Twenty-six specimens were irradiated with the short pulse Er:YAG laser (flashlamp-pumped solid-state laser, Fidelis III, Fotona, Slovenia), with almost square-shaped pulses of adjustable duration, working at $2.94 \mu \mathrm{m}$, fitted with an R02 noncontact handpiece (beam spot size in focus: $0.9 \mathrm{~mm}$ ).
Irradiation was performed using a precision translation platform driven by a stepping motor (ESP301, Newport Corporation, Irvine, California), in such a manner that the laser beam was delivered perpendicular to the dentin surface and at a distance of $7 \mathrm{~mm}$ (focused mode) from the sample's surface. A base, with a fixed dentin sample, was automatically moved $(0.8 \mathrm{~mm} / \mathrm{s})$ based on commands previously established through a computer connected to the scanning device, allowing the irradiation to reach the entire dentin area.

The parameters consider for irradiation were $50 \mu \mathrm{s}$, 2 pulses $/ \mathrm{s}, 80 \mathrm{~mJ}$, and $13.3 \mathrm{~J} / \mathrm{cm}^{2}$. The area to be irradiated was standardized with dimensions of $8 \times 8 \mathrm{~mm}$, covering both sides (sound and eroded) of the sample.

\subsection{Dentin Surface Morphology}

Three samples of each experimental group (dentin hemi-discs) were prepared for analysis under SEM. Samples' preparation for SEM was conducted according to the protocol described by Trevelin et al. $^{25}$ The surface analysis was performed using SEM images (Philipis XL30, The Netherlands) with 1000× and $3000 \times$ magnification.

\subsection{Microshear Bond Strength}

Eighty samples ( $n=20$, dentin hemi-discs) were used, and half of them underwent laser surface treatment following the erosive cycling and prior to the restorative procedure. A 0.05 -mm-thick double-sided adhesive tape piece was placed (Tectape, Manaus, AM, Brazil) on the dentine disc. The tape presented four circular perforations of $1.0 \mathrm{~mm}$ diameter each, delimiting the area of application of the adhesive and the resin cylinder on both SD and ED (two composite resin cylinders for each dentin substrate). These procedures were performed as described by Shimaoka et al. ${ }^{26}$

A self-etching adhesive system (Clearfil SE Bond, Kuraray Medical Inc., Osaka, Japan, Lot. 051 550), used according to the manufacturer's instructions, was applied on the dentin surface. A silicone microtube (Tygon, Norton Performance Plastic Co., Cleveland, Ohio), with an internal diameter of 1.0 and height of $0.5 \mathrm{~mm}$, was used as template for the construction

Table 1 Study design.

46 human molars-46 dentin discs

Microshear bond strength $(n=20)$

40 dentin discs (80 hemi-discs)

40 hemi-discs

40 hemi-discs

Protected surface

20 hemi-discs of SD

Eroded surface

20 hemi-discs of ED

Protected surface

Eroded surface

No surface treatment

20 hemi-discs of SD

20 hemi-discs of ED

Er:YAG laser irradiation

Groups

Surface morphology $(n=3)$
SD

ED

6 dentin discs (12 hemi-discs)
3 hemi-discs of SD 3 hemi-discs of ED

No surface treatment
3 hemi-discs of SD

Er:YAG laser irradiation 
of the composite resin cylinders. After the positioning of four tubes on each dentin disc (two on SD and two on ED), they were filled with composite resin (A3 color, Filtek Z350 XT, 3M ESPE Dental Products, St. Paul, Minnesota, Lot: $14339020560 \mathrm{HB} 004134092$ ) with the aid of a ballpoint probe, also known as a CPI probe (Trinity ${ }^{\circledR}$ Periodontics, Sao Paulo, SP, Brazil). After filling the silicone tubes, the composite resin excesses were removed, with subsequent curing for $20 \mathrm{~s}$ (light-emitting diode source, Radi-lime, SDI Limited, Bayswater, Victoria, Australia) with a power intensity of $1200 \mathrm{~mW} / \mathrm{cm}^{2}$.

After curing, silicone tubes were removed immediately, and all specimens were observed under a stereoscopic lens with $40 \times$ magnification for certifying the absence of defects in the bond interface. The specimens were then stored in artificial saliva ${ }^{27}$ in an incubator at $37^{\circ} \mathrm{C}$ for $24 \mathrm{~h}$, before undergoing the mechanical microshear test. The artificial saliva used for sample storage consisted of $\mathrm{CaCl}_{2}(0.7 \mathrm{mmol} / \mathrm{L})$, $\mathrm{MgCl}_{2} \cdot 6 \mathrm{H}_{2} \mathrm{O}(0.2 \mathrm{mmol} / \mathrm{L}), \mathrm{KH}_{2} \mathrm{PO}_{4}(4.0 \mathrm{mmol} / \mathrm{L}), \mathrm{KCl}$ (30 mmol/L), $\mathrm{NaN}_{3}(0.3 \mathrm{mmol} / \mathrm{L})$, and HEPES buffer (20 mmol/L).

The specimens were submitted to the microshear test on a universal testing machine (Instron 5942, Canton, Massachusetts) ( $1 \mathrm{~mm} / \mathrm{min}$ and $50 \mathrm{~N}$ load). The maximum load values supported by the dentin/restorative material interface were obtained in newtons and later converted to megapascals (MPa).

The results of the bond strength test (average of bond strength obtained from the two resin cylinders of each sample) were analyzed using the statistical software Biostat (AnalystSoft Inc., Walnut, Canada), considering the significance level of $5 \%$. As the distribution of the mean values was normal and homogeneous (Lilliefors normality test), analysis of variance was performed, followed by Tukey's test.

\subsection{Failure Mode}

After completion of the microshear test, all the specimens were analyzed under stereoscopy (Carl Zeiss Citoval 2, Jena, Germany) at $20 \times$ magnification. The failure mode was classified as follows: type I-adhesive; type II-cohesive in dentin; type III-cohesive in composite resin; type IV—mixed.

\section{Results}

\subsection{Dentin Surface Morphology}

Morphological analysis of the specimens showed different characteristics according to the surface treatment.

The SD appeared homogeneous, with grooves caused by the abrasion of the silicon carbide polishing paper and a smear layer covering the whole surface with the obliteration of the dentinal tubules [Figs. 1(a) and 1(b)]. The sound dentin submitted to the laser irradiation (SID) revealed a microretentive pattern with an irregular surface, absence of smear layer, open dentinal tubules, and prominent peritubular dentin [Figs. 1(c) and 1(d)].

The ED samples showed a homogeneous porous surface with large and open dentinal tubules, demineralized peritubular dentin, and a rough intertubular dentin, showing the exposed network of collagen fibers [Figs. 1(e) and 1(f)]. Samples of ED that were treated with the Er:YAG laser (EID) revealed a regular flat surface with obliterated tubules, highlighting a collapse aspect of the collagen fibers [Figs. 1(g) and 1(h)].

\subsection{Microshear Bond Strength}

The microshear bond strength values found for the SD samples, regardless of being irradiated (SID-12.77 $\pm 5.09 \mathrm{~A}$ ) or not (SD-9.76 $\pm 3.39 \mathrm{~B}$ ), were higher than the bond strength values of the eroded samples (ED-5.12 $\pm 1.72 \mathrm{D}$; EID-7.62 $\pm 3.39 \mathrm{C}$ ). Also, the irradiated samples resulted in higher bond strength values than their respective untreated controls (nonirradiated) as described in Fig. 2.

In all groups, the failure mode was predominantly adhesive (between adhesive and dentine), followed by mixed failure and cohesive failure as depicted in Fig. 3.

\section{Discussion}

Without control of the etiologic factors, the loss of dental mineralized structure by erosion can result in dentin exposure. ${ }^{28} \mathrm{In}$ such cases, restorative treatments are indicated to reduce the thermal sensitivity, to prevent injuries to the pulp, and to rehabilitate the contour, function, and aesthetics of the affected elements. ${ }^{29}$ In order to understand the behavior of restorative materials in different dentinal substrates, some authors seek to simulate dental erosion in in vitro protocols, although laboratorial studies present limitations to accurately replicate the in vivo conditions. ${ }^{30}$ In in vitro studies, however, isolated conditions in different tooth substrates exposed to erosive challenges can be investigated under destructive/nondestructive methods.

In the case of dentin exposed to erosive challenges, the acids may remove the plugs that seal the dentinal tubules and demineralize both inter- and peritubular dentin, exposing its organic matrix and increasing the diameter of the tubules. ${ }^{31,32}$ Regarding this aspect, the erosive cycling considered for the present study succeeded in reproducing the characteristics of eroded dentin as seen in SEM images, with open tubules, without perituburar dentin, and having intertubular dentin with a decalcified and fibrous appearance. ${ }^{7,33}$

As adhesive materials must adhere to different surfaces other than those for which they were originally created and tested, studies on ED are mandatory, but adhesion to this substrate has been poorly investigated. Studies have so far found lower bond strength to this substrate compared to $\mathrm{SD},{ }^{6,9,10,34,35}$ corroborating our findings.

The high level of demineralization on ED submitted to etching results in a softened surface layer that, after the penetration of the adhesive, leads to the formation of a thin hybrid layer when compared to an etched SD. These layers are structurally imperfect and contain pores that generate predominantly hydrophilic areas and demineralized zones without resin reinforcement. ${ }^{36}$ These factors can contribute to the low bond strength values found for ED, because resinous monomers' penetration into the demineralized substrate is compromised. ${ }^{34,36,37}$

The irradiated ED showed higher bond strength values than $\mathrm{ED}$, even though their bond strength values were lower than those found for SD (irradiated or not). These findings are consistent with the morphological analysis of the ED submitted to irradiation. The Er:YAG laser irradiation of the ED resulted in a homogeneous demineralized surface with occluded tubules and collapsed collagen fibrils. These characteristics are possibly a result of the residual heat generated during the ablation process, which could have negatively influenced the penetration of the adhesive and the formation of the hybrid layer. ${ }^{38}$ Therefore, treating the surface with the laser parameters tested in the present study was not effective in removing the softened outer dentin layer and improving the adhesion to composite resin. 

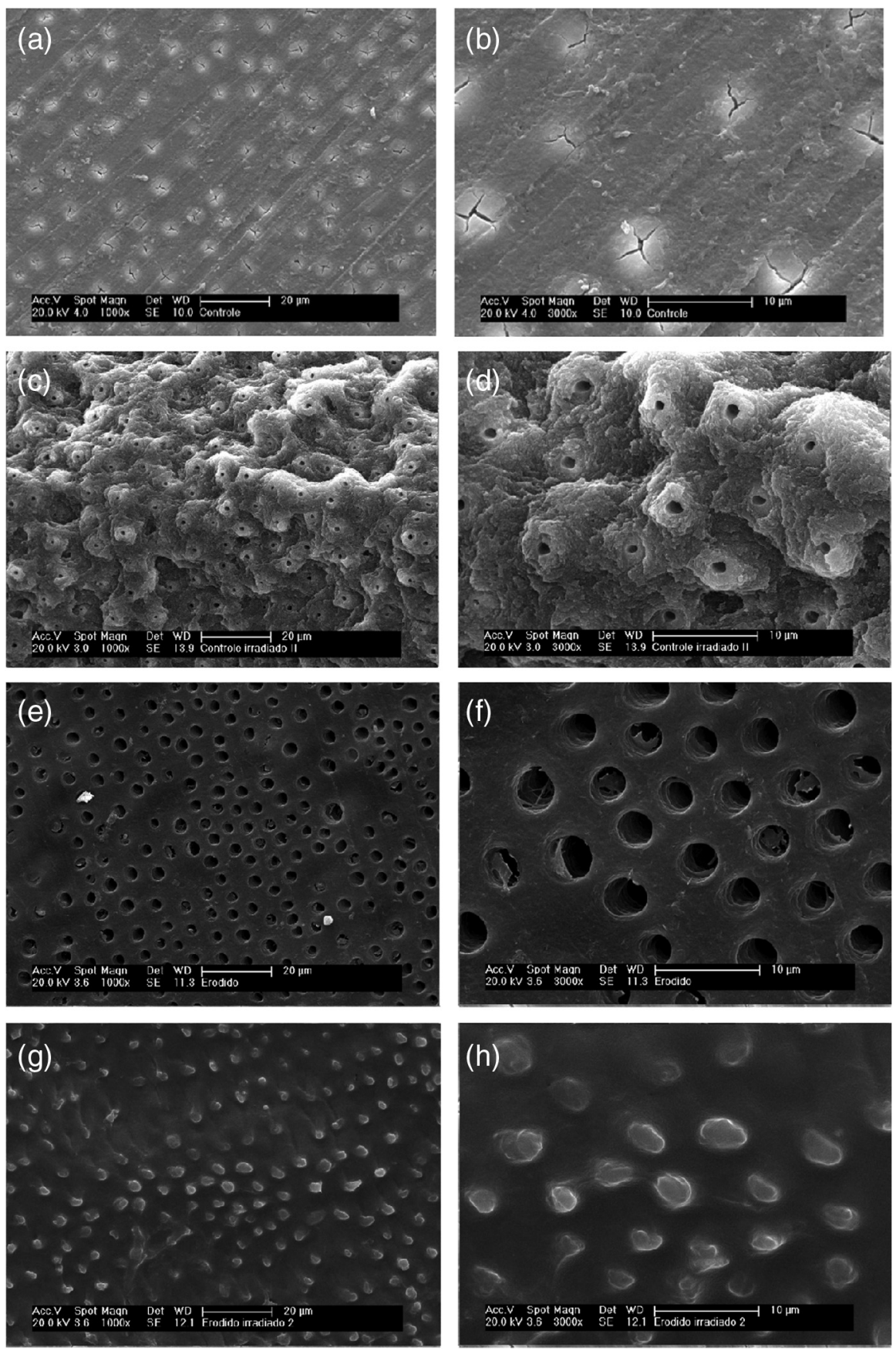

Fig. 1 Electromicrographs obtained by SEM: morphological characteristics of the dentin surfaces after different surface treatments: (a) and (b) SD, (c) and (d) SID, (e) and (f) ED, and (g) and (h) EID.

Ramos et al. ${ }^{10}$ reported that the dentin surface treatment with the Er:YAG laser $\left(60 \mathrm{~mJ}, 2 \mathrm{~Hz}, 0.12 \mathrm{~W}, 19.3 \mathrm{~J} / \mathrm{cm}^{2}, 150\right.$ to $250 \mu \mathrm{s}$ ) was not able to increase the bond strength values to $\mathrm{ED}$ or SD. Their results contrast with our findings that showed that irradiated dentin reached higher bond strength values than those of its nonirradiated counterparts. Even though the energy density considered by Ramos et al..$^{10}$ and the one used in the present study were similar, the higher pulse width used by the authors may have led to thermal damages at the target tissue $^{39}$ and influenced the adhesion to dentin.

Among the tested groups, the irradiated SD was the one that reached the highest shear bond strength values. SEM images showed that, unlike SD (nonirradiated, uniform smear layer, occluded dentinal tubules), SID showed wide opened tubules, irregular surface, and prominent peritubular dentin, creating the microretentive surface that has been suggested by some authors. ${ }^{25,40}$ For Moretto et al., ${ }^{20}$ although the resulting microretentive aspect of the irradiated dentin surface apparently favors the bond strength, it did not actually improve it. The authors reported microcracks on the dentin subsurface and suggested that the decrease in bond strength was due to these changes. According to Esteves-Oliveira et al., ${ }^{13}$ the thermal degradation of collagen may also be a contributing factor to the reduction of bond strength values. 


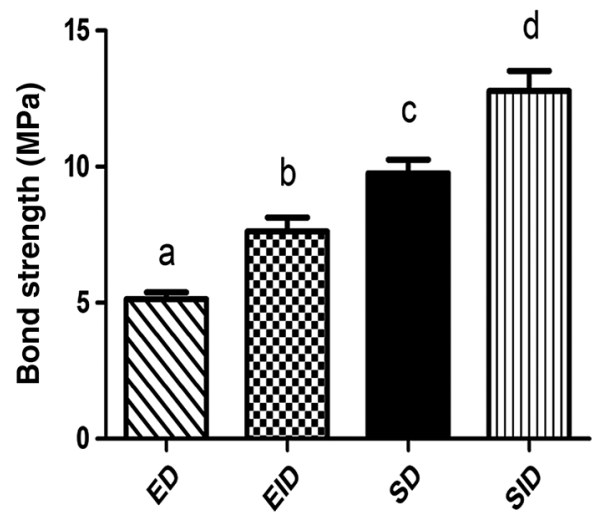

Fig. 2 Microshear bond strength values obtained for all experimental groups.

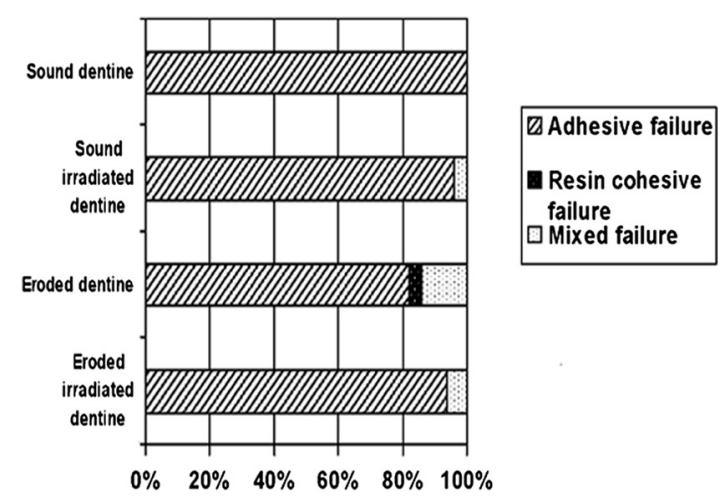

Fig. 3 Prevalence (in percentage) of failure mode for each experimental group.

It is important to highlight that the pulse width of the laser equipment used in previous studies ${ }^{13,20,21,41}$ might have negatively affected the properties of the irradiated tissue, due to the heat diffusion into the inner dentin. ${ }^{39}$ According to Fried et al., ${ }^{42}$ the higher the pulse duration, the higher the residual heat in the irradiated tissue. Bodrumlu et al. ${ }^{43}$ compared the temperature increase during root apicectomy using Er:YAG at three different pulse widths $(50,100$, and $300 \mu \mathrm{s})$ and reported the lowest temperature increase for the $50 \mu \mathrm{s}$ pulse width.

Staninec et al. ${ }^{44}$ suggested that shorter pulse widths, as used in the present study, might minimize the thermal damage to the dentin surface and subsurface. Their results showed that the SD irradiated with a super short pulse Er:YAG laser (35 $\mu$ s) resulted in bond strength similar to the nonirradiated control group. Bahrami et al. ${ }^{45}$ also reported a significant increase in bond strength of the composite resin to dentin when irradiating the substrate with an Er:YAG super short pulse laser $(80 \mathrm{~mJ}$, $10 \mathrm{~Hz}, 50 \mu \mathrm{s}, 12.58 \mathrm{~J} / \mathrm{cm}^{2}$ ).

In the current study, microcracks on the surface of irradiated SD and ED were not expected. ${ }^{46}$ The treatment with the Er:YAG laser (50 $\mu$ s pulse width) with low energy densities appears to be an alternative for tooth pretreatment, without compromising the adhesion to composite resin.

Given the exposures above, we conclude that, regardless of the substrate (SD or ED), the Er:YAG laser with super short pulses favored the dentin bond strength to composite resin. It was the first time that Er:YAG laser was shown to enhance bond strength to ED. Future studies should be conducted in order to assess different laser parameters, the long-term effect of the laser treatment on SD and ED bond strength, and the quality of the bonding interface prior to adhesive procedures.

\section{Conclusion}

The short pulse Er:YAG laser $(50 \mu \mathrm{s})$, when used prior to the restorative treatment, is able to promote positive changes on both SD and ED surface and contribute to the adhesion to composite resin.

\section{References}

1. D. T. Zero, "Etiology of dental erosion-extrinsic factors," Eur. J. Oral Sci. 104(2), 162-177 (1996).

2. A. L. Cavalcanti et al., "Avaliação in vitro do potencial erosivo de bebidas isotônicas," Rev. Bras. Med. Esporte 16(6), 455-458 (2010).

3. M. M. Salas et al., "Diet influenced tooth erosion prevalence in children and adolescents: results of a meta-analysis and meta-regression," $J$. Dent. 43(8), 865-875 (2015).

4. T. S. Carvalho et al., "Consensus report of the European Federation of Conservative Dentistry: erosive tooth wear-diagnosis and management," Clin. Oral Investig. 19(7), 1557-1561 (2015).

5. T. Attin and F. J. Wegehaupt, "Impact of erosive conditions on toothcolored restorative materials," Dent. Mater. 30(1), 43-49 (2014).

6. J. B. Cruz et al., "Eroded dentin does not jeopardize the bond strength of adhesive restorative materials," Braz. Oral Res. 26(4), 306-312 (2012).

7. A. Lussi et al., "Dental erosion- an overview with emphasis on chemical and histopathological aspects," Caries Res. 45(Suppl 1), 2-12 (2011).

8. C. Ganss et al., "Effects of toothbrushing on eroded dentine," Eur. J. Oral Sci. 115(5), 390-396 (2007).

9. B. Zimmerli et al., "Long-term bonding to eroded dentin requires superficial bur preparation," Clin. Oral Investig. 16(5), 1451-1461 (2012).

10. T. M. Ramos et al., "Effects of Er:YAG and Er, Cr:YSGG laser irradiation on the adhesion to eroded dentin," Lasers Med. Sci. 30(1), 17-26 (2015).

11. R. Hibst and U. Keller, "Experimental studies of the application of Er: YAG laser on dental hard substances: I. Measurement of the ablation rate," Lasers Surg. Med. 9(4), 338-344 (1989).

12. T. M. Ramos et al., "Microtensile bond strength analysis of adhesive systems to Er:YAG and Er, Cr:YSGG laser-treated dentin," Lasers Med. Sci. 29(2), 565-573 (2014).

13. M. Esteves-Oliveira et al., "Bond strength of self-etching primer to bur cut, Er, Cr:YSGG, and Er:YAG lased dental surfaces," Photomed. Laser Surg. 25(5), 373-380 (2007).

14. A. Aoki et al., "Comparison between Er:YAG laser and conventional technique for root caries treatment in vitro," J. Dent. Res. 77(6), 1404-1414 (1998).

15. A. Baraba et al., "Ablative potential of four different pulses of Er:YAG lasers and low-speed hand piece," Photomed. Laser Surg. 30(6), 301307 (2012).

16. A. Baraba et al., "Influence of different pulse durations of Er:YAG laser based on variable square pulse technology on microtensile bond strength of a self-etch adhesive to dentin," Photomed. Laser Surg. 31(3), 116-124 (2013).

17. M. Lukac, N. M. Primc, and S. Pirnat, "Quantum square pulse Er:YAG lasers for fast and precise hard dental tissue preparation," J. Laser Health Acad. 2012(1), 14-21 (2012).

18. L. Ceballos et al., "Bonding to Er:YAG laser treated dentin," J. Dent. Res. 81(2), 119-122 (2002).

19. L. Manhães et al., "Influence of Er:YAG laser surface treatment and primer application methods on microtensile bond strength self-etching systems," Photomed. Laser Surg. 23(3), 304-312 (2005).

20. S. G. Moretto et al., "Effects of ultramorphological changes on adhesion to lased dentin-scanning electron microscopy and transmission electron microscopy analysis," Microsc. Res. Tech. 74(8), 720-726 (2011).

21. M. Esteves-Oliveira et al., "Influence of the additional Er:YAG laser conditioning step on the microleakage of class $\mathrm{V}$ restorations," $J$. Biomed. Mater. Res. B Appl. Biomater. 87(2), 538-543 (2008). 
22. E. Firat, S. Gurgan, and N. Gutknech, "Microtensile bond strength of an etch-and-rinse adhesive to enamel and dentin after Er:YAG laser pretreatment with different pulse durations," Lasers Med. Sci. 27(1), 15-21 (2012).

23. C. Ganss et al., "Effectiveness of two fluoridation measures on erosion progression in human enamel and dentine in vitro," Caries Res. 35(5), 325-330 (2001).

24. D. T. Zero et al., "Comparison of the iodide permeability test, the surface microhardness test, and mineral dissolution of bovine enamel following acid challenge," Caries Res. 24(3), 181-188 (1990).

25. L. T. Trevelin et al., "Effect of super short pulse Er:YAG laser on human dentin-scanning electron microscopy analysis," Microsc. Res. Tech. 78(6), 472-478 (2015).

26. A. M. Shimaoka et al., "The importance of adhesive area delimitation in a microshear bond strength experimental design," J. Adhes. Dent. 13(4), 307-314 (2011).

27. D. H. Pashley et al., "Collagen degradation by host-derived enzymes during aging," J. Dent. Res. 83(3), 216-221 (2004).

28. T. Jaeggi, A. Gruninger, and A. Lussi, "Restorative therapy of erosion," Monogr. Oral Sci. 21, 200-214 (2006).

29. X. Wang and A. Lussi, "Assessment and management of dental erosion," Dent. Clin. North Am. 54(3), 565-578 (2010).

30. A. Young and L. M. Tenuta, "Initial erosion models," Caries Res. 45(Suppl 1), 33-42 (2011).

31. C. Prati et al., "Permeability and morphology of dentin after erosion induced by acidic drinks," J. Periodontol. 74(4), 428-436 (2003).

32. S. Wongkhantee et al., "Effect of acidic food and drinks on surface hardness of enamel, dentine, and tooth-coloured filling materials," J. Dent. 34(3), 214-220 (2006).

33. G. W. Marshall, Jr et al., "Effect of citric acid concentration on dentin demineralization, dehydration, and rehydration: atomic force microscopy study," J. Biomed. Mater. Res. 42(4), 500-507 (1998).

34. J. B. Cruz et al., "Bonding stability of adhesive systems to eroded dentin," Braz. Oral Res. 29, S1806 (2015).

35. L. F. Francisconi-dos-Rios et al., "Role of chlorhexidine in bond strength to artificially eroded dentin over time," J. Adhes. Dent. 17(2), 133-139 (2015).

36. Y. Wang and P. Spencer, "Effect of acid etching time and technique on interfacial characteristics of the adhesive-dentin bond using differential staining," Eur. J. Oral Sci. 112(3), 293-299 (2004).

37. F. R. Tay and D. H. Pashley, "Resin bonding to cervical sclerotic dentin: a review," J. Dent. 32(3), 173-196 (2004).

38. L. Ceballos et al., "Microleakege of composite restorations after acid Er: YAG laser cavity treatments," Dent. Mater. 17(4), 340-346 (2001).

39. B. Majaron et al., "Heat diffusion and debris screening in Er:YAG laser ablation of hard biological tissues," Appl. Phys. B 66(4), 479-487 (1998).

40. S. R. Visuri et al., "Shear strength of composite bonded to Er:YAG laser prepared dentine," J. Dent. Res. 75(1), 599-605 (1996).

41. A. C. Aranha et al., "Analysis of the interfacial micromorphology of adhesive systems in cavities prepared with Er, Cr:YSGG, Er:YAG laser and bur," Microsc. Res. Tech. 70(8), 745-751 (2007).
42. D. Fried, J. Ragadio, and A. Champion, "Residual heat deposition in dental enamel during IR laser ablation at 2.79, 2.94, 9.6 and 10.6 lm," Lasers Surg. Med. 29(3), 221-229 (2001).

43. E. Bodrumlu et al., "Temperature variation during apicectomy with Er: YAG laser," Photomed. Laser Surg. 30(8), 425-428 (2012).

44. M. Staninec et al., "Adhesion of composite to enamel and dentin surfaces irradiated by IR laser pulses of 0.5-35 micros duration," J. Biomed. Mater. Res. 79B(1), 199-201 (2006).

45. B. Bahrami et al., "Effect of low fluency dentin conditioning on tensile bond strength of composite bonded to Er:YAG laser-prepared dentin: a preliminary study," Lasers Med. Sci. 26(2), 187-191 (2011).

46. W. Raucci-Neto et al., "Thermal effects and morphological aspects of varying Er:YAG laser energy on demineralized dentin removal: an in vitro study," Lasers Med. Sci. 30(4), 1231-1236 (2015).

Maria Cecília Pereira Cersosimo graduated from the Dental School of the University of São Paulo in 2012 and received her master's degree in operative dentistry from the School of Dentistry at the University of Sao Paulo (FOUSP) in 2016.

Adriana Bona Matos received her $\mathrm{PhD}$ in operative dentistry from the School of Dentistry at the University of Sao Paulo (FOUSP) in 1996. She is currently a full-time professor at the same institution. Her research activity is focused on the effect of substrate modification on in vitro bonding to dentine, including laser irradiated hard tissues and caries affected dentine.

Roberta Souza D'Almeida Couto received her degree of doctorate in dentistry from the University of São Paulo (USP) in 2013. Recently, she completed a postdoctoral fellowship in dentistry at USP. She is a professor at the Federal University of Pará (UFPA), and her research activity is focused on biomaterials for dental use.

Márcia Martins Marques received her $\mathrm{PhD}$ in oral pathology from the School of Dentistry at the University of Sao Paulo (FOUSP) in 1991. She is currently a full professor at the same institution. Her research activity is focused on the effect of laser photobiomodulation in tissue regeneration.

Patricia Moreira de Freitas graduated from the Dental School of the State University of Campinas in 2000. She concluded her master's degree program (operative dentistry) at the Dental School of the State University of Campinas in 2003 and her $\mathrm{PhD}$ (operative dentistry) at the School of Dentistry at the University of São Paulo in 2005. She was a visiting professor at the University of California in San Francisco in 2005. She is currently an associate professor at the Department of Operative Dentistry in the School of Dentistry at the University of São Paulo and co-chairman of the Special Laboratory of Lasers in Dentistry. 